\title{
Umsetzung der neuen Approbationsordnung für Ärzte im Querschnittsbereich „Rehabilitation, Physikalische Medizin und Naturheilverfahren" an den medizi- nischen Fakultäten in Deutschland
}

Implementation of the New Federal Medical Licensing Regulations in the Interdisciplinary Subject „Rehabilitation, Physical Medicine, Naturopathic Treatment“ by the German Medical Faculties

\section{Zusammenfassung}

Mit der Einführung des Querschnittsbereiches „Rehabilitation, Physikalische Medizin, Naturheilverfahren“(QSB RPMN) im Rahmen der 9. Revision der Approbationsordnung für Ärzte ergeben sich für die Lehre der Rehabilitation neue Chancen und Herausforderungen. Um die Umsetzung der neuen Approbationsordnung im QSB RPMN an den einzelnen medizinischen Fakultäten zu untersuchen, wurde bei ihnen eine schriftliche Befragung durchgeführt. Für die Realisierung neuer Formen und Verfahren der Lehre im QSB RPMN wurden verschiedene Indikatoren bei der Befragung untersucht. Von der Hälfte der Fakultäten werden Blockveranstaltungen vorgesehen. Vorlesungen bilden die dominierende Veranstaltungsform mit durchschnittlich mehr als der Hälfte der für den QSB RPMN verfügbaren Zeit. Für den Leistungsnachweis werden in $83 \%$ schriftliche Prüfungen durchgeführt, gegenüber den nur in $17 \%$ angegebenen Objective Structured Clinical Examinations (OSCEs). Bei unterschiedlicher Ausgestaltung des QSB RPMN in den medizinischen Fakultäten besteht vielfach noch Raum für Verbesserungen im Sinne der praxis- und patientennahen Ausbildung, die in der Approbationsordnung intendiert ist. Allerdings werden trotz gestiegener Anforderungen kaum zusätzliche Sach- oder Personalmittel zur Verfügung gestellt. Wichtige zukünftige Impulse für die Weiterentwicklung der Ausbildungsangebote sind von der intrauniversitären leistungsbezogenen Mittelvergabe für die Lehre und der interuniversitären Profilierung bei der ärztlichen Ausbildung

\section{Abstract}

The introduction of the new interdisciplinary subject „Rehabilitation, Physical Medicine, Naturopathic Treatment“ based on the $9^{\text {th }}$ revision of the Federal Medical Licensing Regulations (Approbationsordnung für Ärzte) implies new opportunities and challenges for teaching. In order to investigate the transfer of the new subject to teaching practise, a written questionnaire was sent to all German medical faculties. With regard to the implementation of new teaching structures and procedures in the subject, a range of different indicators was examined. $50 \%$ of the medical faculties have been planning for comprehensive teaching sessions as compared to distribution over the whole semester. Lectures predominate with an average of more than half of the teaching time available for the subject. For examination purposes written tests are used in $83 \%$, compared to only $17 \%$ Objective Structured Clinical Examinations. Considering the diversity among the medical faculties, there is room for improvement in many universities concerning more patient- and practice-oriented medical training as intended by the Federal Medical Licensing Regulations. However, in spite of increased demands, supplementary funds for additional equipment or manpower are rarely provided for. In the future, enhanced allocation of resources based on performance-related evaluation within the medical faculties and teaching competition between universities may provide significant stimuli for advances in medical training also in the new interdisciplinary subject. Further improvement 
auch im QSB RPMN zu erwarten. Eine Optimierung der Rahmenbedingungen ist zu fordern, um die Chancen der neuen Approbationsordnung im QSB RPMN auszuschöpfen.

\section{Schlüsselwörter}

Approbationsordnung $\cdot$ Lehre $\cdot$ Rehabilitation $\cdot$ Physikalische Medizin · Naturheilverfahren · Studium Humanmedizin of general teaching conditions is necessary in order to utilize all the opportunities offered by the new Federal Medical Regulations.

\section{Key words}

Federal Medical Licensing Regulations - rehabilitation · physical medicine $\cdot$ naturopathic treatment $\cdot$ teaching $\cdot$ medicine

\section{Einleitung}

Mit der neuen, am 1.10.2003 in Kraft getretenen 9. Revision der Approbationsordnung für Ärzte [1] wurde auch der obligatorische Querschnittsbereich „Rehabilitation, Physikalische Medizin, Naturheilverfahren" (QSB RPMN) eingeführt. Erstmalig ist damit in allen medizinischen Fakultäten in Deutschland die Basis dafür geschaffen, bereits im Medizinstudium die wesentlichen Inhalte und Konzepte der Rehabilitation und die für jeden Arzt relevanten Grundlagenkenntnisse zu rehabilitationsbezogenen Themenbereichen entsprechend dem Konzept der Internationalen Klassifikation der Funktionsfähigkeit, Behinderung und Gesundheit (ICF) der Weltgesundheitsorganisation sowie den Erfordernissen des Neunten Buches Sozialgesetzbuch (SGB IX) den Studierenden patienten- und praxisbezogen zu vermitteln. Gleichzeitig wird im QSB RPMN besonders die Relevanz der interdisziplinären Kooperation mit Einschluss der psychosozialen Fächer verdeutlicht [2]. Für die Fakultäten ergeben sich mit der Lehrverpflichtung zur Rehabilitation und der Einbettung in den Querschnittsbereich eine Reihe von neuen inhaltlichen und organisatorischen Herausforderungen und Chancen. Da bisher keine konkreten, anerkannten Lehrstandards für den QSB RPMN existierten, wurden Empfehlungen zu Lernzielen von der Kommission „Aus-, Fort- und Weiterbildung“ der Deutschen Gesellschaft für Rehabilitationswissenschaften (DGRW) gemeinsam mit der Initiativgruppe „Ausbildung“ der Deutschen Gesellschaft für Physikalische Medizin und Rehabilitation (DGPMR) erarbeitet [3].

Die konkrete Ausgestaltung des QSB RPMN obliegt nun den einzelnen Universitäten und Hochschulen. Dazu gehört auch die Anwendung moderner Lehr- und Prüfungsmethoden, die bereits mit Erfolg u.a. in Reformstudiengängen eingesetzt werden [4], wie problemorientiertes Lernen (POL) [5], Computer Based Teaching (CBT) [6] oder Objective Structured Clinical Examinations (OSCEs) [7].

Es ist zu vermuten, dass die Fakultäten den gegebenen Spielraum in Abhängigkeit von den vorhandenen Schwerpunkten, Spezialisierungen und Ressourcen in unterschiedlicher Weise nutzen. Um die Umsetzung der neuen Approbationsordnung im QSB RPMN an den deutschen medizinischen Fakultäten zu untersuchen, wurde bei ihnen im Auftrag der Kommission „Aus-, Fortund Weiterbildung" und mit Förderung der DGRW von Januar bis März 2004 eine schriftliche Befragung durchgeführt.

\section{Methoden}

Im ersten Schritt wurden die Studiendekane aller 37 medizinischen Fakultäten in Deutschland schriftlich nach den Beauftrag- ten für den QSB RPMN befragt. Im zweiten Schritt erhielten diese Beauftragten einen anonym auszufüllenden standardisierten Fragebogen. Wenn kein Beauftragter zu ermitteln war, wurde der Fragebogen an den Studiendekan versandt. Dem Brief lagen ein einführendes Begleitschreiben sowie ein frankierter Rückumschlag bei. Nach sechs Wochen wurde bei fehlender Antwort ein Erinnerungsschreiben verschickt. Der Fragebogen beinhaltete Fragen zur Anzahl der Studierenden, zur zeitlichen Struktur des Studiums, zum Zeitpunkt der Lehre für den QSB RPMN, zu Orten, Formen und Zeitanteilen der Lehrveranstaltungen, zu Zeitanteilen der drei Unterbereiche des QSB RPMN, zu Lehr- und Prüfungsmethoden sowie zu Problemen und positiven Aspekten der Umsetzung.

\section{Ergebnisse}

\section{Beauftragte für den QSB RPMN}

32 der 37 Fakultäten (86\%) haben die schriftliche Anfrage nach Beauftragten für den QSB RPMN beantwortet. 30 Fakultäten (79\%) konnten Beauftragte benennen (Tab.1). Zirka ein Drittel der Beauftragten $(n=11)$ repräsentiert eines der Fächer entsprechend der Bezeichnung im QSB RPMN, am häufigsten die Physikalische Medizin. Die Mehrheit ( $\mathrm{n}=17)$ kommt aus einem anderen klinischen Fach, am häufigsten aus der Orthopädie. Zwei Fakultäten hatten zum Zeitpunkt der Auswertung des Fragebogens noch keinen Beauftragten ernannt.

\section{Allgemeine Studienangaben}

24 der 37 Fakultäten (65\%) beantworteten den im 2. Schritt versandten Fragebogen. Bei 75\% wird das Studienjahr in Semester,

Tab. 1 Institute bzw. Kliniken der Beauftragten für den QSB RPMN (Angaben von 32 Fakultäten mit Rückmeldungen)

1. Klinik/Institut entsprechend Unterbereichen des QSB RPMN $(n=11)$
Physikalische Medizin bzw. Physikalische Medizin/Rehabilitation $(n=6)$
Naturheilkunde $(n=3)$
Rehabilitationsmedizin $(n=2)$
2. klinische Fächer $(n=17)$
Orthopädie bzw. Orthopädie/Rheumatologie $(n=6)$
Allgemeinmedizin $(n=3)$
Chirurgie/Unfall- und Wiederherstellungschirurgie $(n=2)$
Psychiatrie $(n=2)$
Sozialmedizin bzw. Sozialmedizin und Qualitätsmanagement $(n=2)$
Arbeitsmedizin $(n=1)$
Neurologie ( $n=1)$
3. vorklinische Fächer $(n=2)$
medizinische Psychologie bzw. Psychologie/Musiktherapie $(n=2)$
4. Beauftragter (noch) nicht benannt $(n=2)$




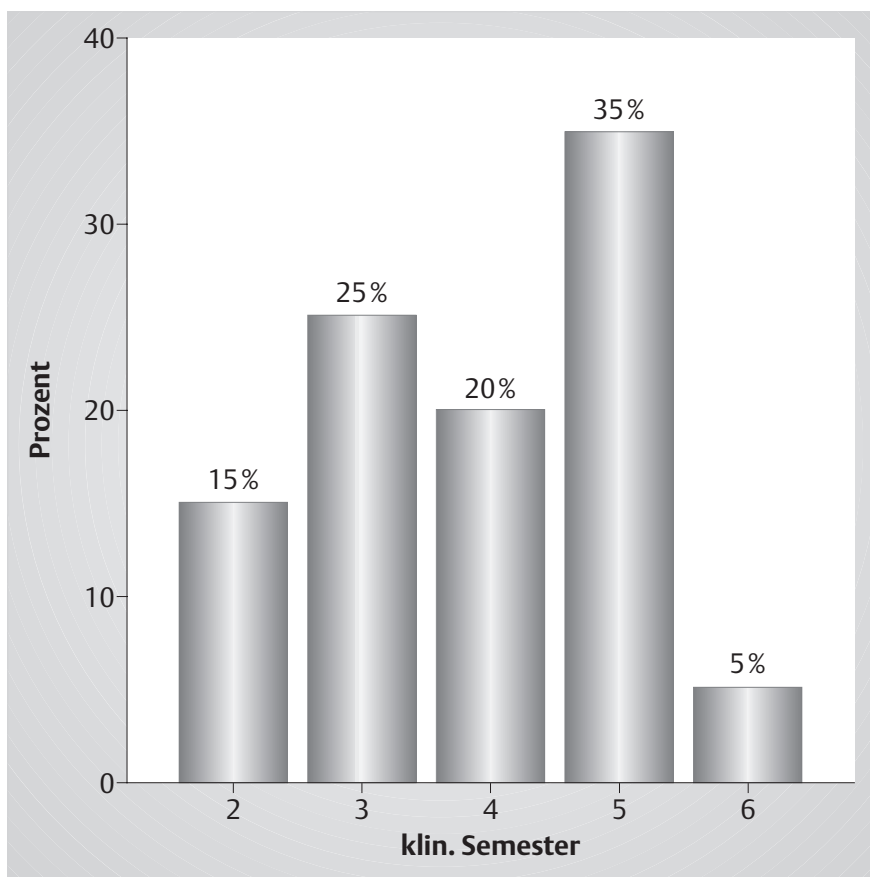

Abb. 1 Zeitpunkt des Lehrangebots zum Querschnittsbereich während des klinischen Studienabschnitts.

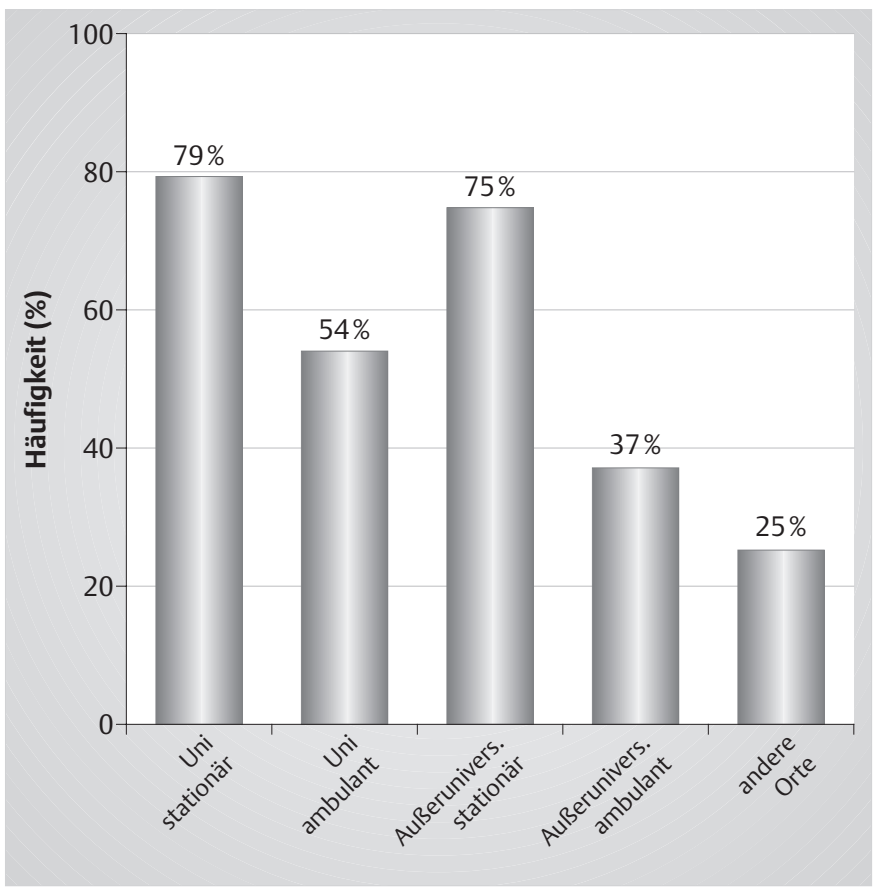

Abb. 2 Veranstaltungsorte für die Lehre.

bei $8 \%$ in Tertiale und bei $17 \%$ nicht weiter unterteilt. Im Mittel gehen die Hochschulen von 240 Studenten pro Jahr aus (min. 42 bis max. 600). Nur drei Einrichtungen schätzten die Zahl der zu erwartenden Studenten auf 450 oder mehr.

\section{Lehrveranstaltungen im QSB RPMN}

Am häufigsten (35\%) werden die Lehrveranstaltungen des QSB RPMN im 5. klinischen Semester angeboten (Spanne 2. -6. klinisches Semester) (Abb.1).
Tab. 2 Vorgesehene Veranstaltungsformen und Stundenzahl im QSB RPMN

\begin{tabular}{llllll}
\hline Vorlesung & Seminar & $\begin{array}{l}\text { praktische } \\
\text { Übungen }\end{array}$ & $\begin{array}{l}\text { Studien- } \\
\text { gruppe }\end{array}$ & gesamt \\
& $\begin{array}{l}\text { Mittel der } 19 \mathrm{~h} \\
\begin{array}{l}\text { Stundenzahl } \\
\text { Spanne }\end{array}\end{array} \quad 10 \mathrm{~h}$ & $7 \mathrm{~h}$ & $1 \mathrm{~h}$ & $37 \mathrm{~h}$ \\
\hline
\end{tabular}

Tab. 3 Zeitliche Aufteilung der Unterbereiche Rehabilitation (nur „übergreifende“ Themen wie ICF, Reha-Formen), Physikalische Medizin (ohne „übergreifende“ Aspekte der Rehabilitation) sowie klassische und alternative Naturheilverfahren

\begin{tabular}{lllll}
\hline & Rehabilitation & $\begin{array}{l}\text { Physikalische } \\
\text { Medizin }\end{array}$ & $\begin{array}{l}\text { klassische } \\
\text { Naturheil- } \\
\text { verfahren }\end{array}$ & $\begin{array}{l}\text { alternative } \\
\text { Naturheil- } \\
\text { verfahren }\end{array}$ \\
\hline Mittelwert & $40 \%$ & $33 \%$ & $18 \%$ & $9 \%$ \\
\hline Spanne & $20-95 \%$ & $5-60 \%$ & $0-50 \%$ & $0-20 \%$ \\
\hline
\end{tabular}

Bei 33\% der Befragten werden die Lehrveranstaltungen ausschließlich in (Einzel-)Stunden über mehrere Wochen bzw. Monate über das Semester bzw. Studienjahr verteilt. 50\% der Fakultäten planen alternativ oder zusätzlich die Durchführung von Blockveranstaltungen. Lediglich 13\% der Fakultäten wollen ihre Veranstaltungen durchgängig in Blockform organisieren.

Die Veranstaltungsorte sind weit überwiegend stationär sowohl in universitären als auch außeruniversitären Einrichtungen, seltener ambulant oder an anderer Stelle (z. B. vertragsärztliche Praxen, Krankengymnastikschule) (Abb. 2).

Die Lehrveranstaltungen des QSB RPMN mit einer mittleren Gesamtdauer von 37 Stunden werden deutlich von Vorlesungen mit im Mittel 20 Stunden dominiert, während praktische Übungen durchschnittlich nur 7 Stunden einnehmen (Tab. 2). Die Unterschiede zwischen den Fakultäten sind sehr groß und z.T. durch Besonderheiten bedingt. So ist das Maximum von 105 Stunden des Gesamtlehrangebots durch die Longitudinalveranstaltungen über mehrere Studienjahre zu erklären.

Der größte Zeitanteil im QSB RPMN (im Mittel 40\%) wurde für die Rehabilitation (nur „übergreifende“ Themen der Rehabilitation wie ICF, Reha-Formen) geschätzt, gefolgt von der Physikalischen Medizin (ohne „übergreifende“ Aspekte der Rehabilitation) mit 33\%. Der Anteil der klassischen Naturheilverfahren wurde im Mittel mit 18\% und der Teil für alternative Naturheilverfahren mit durchschnittlich $9 \%$ angegeben.

\section{Lehr- und Prüfungsmethoden}

Multimediale Präsentationen und strukturierte Fallbeispiele sind mit jeweils $92 \%$ die am häufigsten eingesetzten Lehrmethoden, gefolgt von eigenständigem Erarbeiten von Inhalten (67\%), problemorientiertem Lernen (54\%) sowie anderen Methoden (17\%) wie Computer Based Teaching, Fallvorstellungen, Leitsymptomvorlesung, Eigenerfahrung (Mehrfachnennungen möglich). 
Im Vordergrund der im QSB RPMN zu erbringenden Leistungsnachweise steht die schriftliche Prüfung (83\%). Selten werden mündliche Prüfungen (21\%), OSCEs (17\%) und andere Leistungsnachweise wie mündliche Beteiligung, Referat etc. (4\%) genannt (Mehrfachnennungen möglich).

\section{Probleme und positive Aspekte der Umsetzung}

An zahlreichen Standorten wurden Probleme bei der Umsetzung des QSB RPMN genannt, die hauptsächlich finanzieller und personeller Natur sind. Lediglich einer der Befragten gab an, zusätzliche Sachmittel (2000 Euro ohne Angabe eines Zeitrahmens) zu erhalten. Zwei weiteren Befragten waren zusätzliche Personalmittel für Lehraufträge bzw. wissenschaftliche Hilfskräfte bewilligt worden.

Als weitere Schwierigkeiten wurden angeführt: Bereitstellung von Patienten für Fallvorstellungen, Mangel an vor Ort vorhandenen Lehrmöglichkeiten und Reha-Einrichtungen, Zusammenarbeit und Koordination der Fachvertreter von Rehabilitation, Physikalischer Medizin und Naturheilverfahren, thematische Heterogenität des QSB RPMN, Koordination mit dem Gesamtlehrplan sowie zu geringe Vertretung der Physikalischen Medizin und Naturheilverfahren.

Die sich aus der Umsetzung der neuen Approbationsordnung ergebenden positiven Aspekte umfassen die Gewinnung des studentischen Interesses für die im QSB RPMN repräsentierten Fächer (auch i.S. von Nachwuchsrekrutierung), die Darstellung von Rehabilitation als wichtigen und elementaren Bestandteil der medizinischen Versorgung, die Erhöhung des Stellenwertes der Rehabilitation in der ärztlichen Ausbildung, in der praktischen Tätigkeit und in der Fakultät sowie die Stärkung der interdisziplinären Kooperation.

\section{Diskussion}

Die neue Approbationsordnung für Ärzte wurde unter anderem mit dem Ziel einer verstärkt praxis- und patientenorientierten sowie fächerübergreifenden Ausbildung konzipiert. Damit sind wesentliche Ziele der seit mehreren Jahren bestehenden Reformstudiengänge der Humanmedizin [4] auch für den Regelstudiengang deutlicher formuliert. Eine erfolgreiche inhaltliche Ausgestaltung der Lehre im QSB RPMN nach diesen Zielen und den Maßgaben der neuen Approbationsordnung hängt wesentlich davon ab, inwieweit geeignete strukturelle und organisatorische Rahmenbedingungen hierfür vorhanden sind oder geschaffen werden können. Für den Lehrbereich der Rehabilitation ist in diesem Zusammenhang die Stärkung der rehabilitationswissenschaftlichen Infrastruktur an den Universitäten in Form von Stiftungsprofessuren und/oder Querschnittseinrichtungen von Bedeutung, die in den acht regionalen Forschungsverbünden im Förderschwerpunkt Rehabilitationswissenschaften geschaffen wurden [8]. In mehreren Universitäten wird die Organisation der Lehre im QSB RPMN durch Beauftragte wahrgenommen, die vor allem aus Kliniken und Instituten stammen, in denen zumindest nach ihrer Bezeichnung vor allem die anderen beiden Schwerpunkte des QSB, Physikalische Medizin und Naturheilverfahren bzw. Naturheilkunde, vertreten werden. Unter den anderen klinischen Fächern dominiert die Orthopädie bei der Lehror- ganisation, was sowohl die häufige universitäre Mitvertretung der Physikalischen Medizin durch dieses Fach als auch die herausragende Bedeutung der muskuloskeletalen Erkrankungen bei den Rehabilitationsmaßnahmen reflektiert [9]. Inwieweit Einrichtungen der regionalen rehabilitationswissenschaftlichen Forschungsverbünde oder deren Folgestrukturen konkret in die Gestaltung der Lehre im QSB RPMN einbezogen sind, soll in einer weiteren Befragung zur Entwicklung der Lehre untersucht werden.

Für die Realisierung neuer Formen und Verfahren der Lehre im QSB RPMN wurden verschiedene Indikatoren bei der Befragung untersucht. Auch wenn 83 \% der Universitäten die klassische Verteilung des Lehrangebots über das Semester bzw. Studienjahr angeben, werden von der Hälfte (meistens zusätzliche) Blockveranstaltungen vorgesehen. Die Behandlung des Lehrstoffs in diesen Einheiten bietet besondere Chancen der komprimierten $\mathrm{Zu}$ sammenführung verschiedener Inhalte, Professionen, Fachdisziplinen sowie Lehr- und Lernformen.

Vorlesungen sind die überwiegende Veranstaltungsform mit durchschnittlich mehr als der Hälfte der für den QSB RPMN verfügbaren Zeit, gefolgt von Seminaren mit Gruppengrößen bis 20 Studierenden gemäß der Approbationsordnung. Die für neue Lehr- und Lernmethoden besonders geeigneten Kleingruppen bei den praktischen Übungen und den Studiengruppen umfassen im Mittel ca. ein Viertel der Gesamtzeit; einige Fakultäten bieten sie allerdings gar nicht an. Eher konservativ sind auch die Verfahren zum Leistungsnachweis mit 83\% schriftlichen Prüfungen gegenüber den neueren, aber aufwändigen OSCEs, die nur $17 \%$ der Fakultäten praktizieren.

Diese Beispiele verdeutlichen, dass grundsätzlich noch Raum für Verbesserungen der Lehre in Richtung der modernen praxis- und patientennahen Ausbildung zu bestehen scheint, die in der Approbationsordnung intendiert ist. In der universitären Praxis stößt diese Forderung aber auf klare Grenzen. Den Fakultäten werden bis auf kaum bedeutsame Ausnahmen keine zusätzlichen Sach- oder Personalmittel zur Verfügung gestellt, sodass die gestiegenen Anforderungen im neuen QSB RPMN mit dem gleichen oder aus anderen Gründen des universitären Sparzwangs sogar mit reduziertem Personal bewältigt werden müssen. Hinzu kommt die gegenüber den Forschungsleistungen bisher gar nicht oder selten vollzogene budgetrelevante Erfassung der Lehrqualität und -quantität in den meisten Fakultäten mit entsprechenden Konsequenzen für die universitäre Leistungsorientierung und Wertschätzung. Daraus sind die von zahlreichen Einrichtungen bevorzugten, wenig personalintensiven Lehrangebote und Leistungsnachweise verständlich. Wichtige Impulse für die Weiterentwicklung der Ausbildungsangebote sind in Zukunft von der intrauniversitären leistungsbezogenen Mittelvergabe für die Lehre zu erwarten, die in einem wirksamen Verhältnis zum forschungsbezogenen Budget stehen muss, und von der interuniversitären Profilierung bei der ärztlichen Ausbildung in der zunehmenden Konkurrenz, auch um die Studierenden. Eine Optimierung der Rahmenbedingungen ist dringend $\mathrm{zu}$ fordern, um die Chancen, die die neue Approbationsordnung im QSB RPMN bietet, auszuschöpfen. 


\section{Danksagung}

Die Autoren danken den Mitgliedern der Kommission „Aus-, Fort- und Weiterbildung“ der Deutschen Gesellschaft für Rehabilitationswissenschaften, Herrn Dr. M. Gülich, Frau B. Lampe, Herrn Dr. M. Morfeld, Frau Dr. S. R. S. Schwarzkopf, und dem Leiter der Initiativgruppe „Ausbildung“ der Deutschen Gesellschaft für Physikalische Medizin und Rehabilitation, Herrn Prof. Dr. U. Smolenski, für die Anregungen bei der Erstellung des Fragebogens und der Diskussion der Ergebnisse. Unser Dank gilt weiterhin Herrn Carsten Ranft für seine Unterstützung bei der Datenerhebung und -aufbereitung.

\section{Literatur}

${ }^{1}$ Approbationsordnung für Ärzte, 9. Revision. Bundesgesetzblatt Teil I 2002; 44: 2405-2435

${ }^{2}$ Strauß B, Köllner V. Die neue Approbationsordnung: Eine Chance für die psychosozialen Fächer. Psychotherapie Psychosomatik Medizinische Psychologie 2003; 53: $43-46$

${ }^{3}$ Mau W, Gülich M, Gutenbrunner C, Lampe B, Morfeld M, Schwarzkopf SR, Smolenski UC. Lernziele im Querschnittsbereich Rehabilitation, Physikalische Medizin und Naturheilverfahren nach der 9. Revision der Approbationsordnung der Ärzte. Gemeinsame Empfehlung der Deutschen Gesellschaft für Rehabilitationswissenschaften und der Deutschen Gesellschaft für Physikalische Medizin und Rehabilitation. Rehabilitation 2004; 43: 337 - 347

${ }^{4}$ Burger W, Dudenhausen JW, Kiessling C, Scheffner D, Wilke A. Reform des Medizinstudiums. Positive Erfahrungen an der Charité Berlin. Dtsch Ärztebl 2003; 100: A 686-689

${ }^{5}$ Barrows HS, Tamblyn RM. Problem-based learning: an approach to medical education. Springer Series on Medical Education. New York: Springer Publishing Company, 1980

${ }^{6}$ Issing L, Klimsa P. Information und Lernen mit Multimedia. Weinheim: Psychologie-Verlags-Union, 1995

${ }^{7}$ Harden RM, Stevenson M, Downie WW, Wilson GM. Assessment of clinical competence using Objective Structured Examination. BMJ $1975 ; 1: 447-451$

8 Zwingmann C, Buschmann-Steinhage R, Gerwinn H, Klosterhuis H. Förderschwerpunkt Rehabilitationswissenschaften: Ergebnisse - Umsetzung - Erfolge und Perspektiven. Rehabilitation 2004; 43: 260270

${ }^{9}$ Verband Deutscher Rentenversicherungsträger. VDR Statistik Rehabilitation des Jahres 2003. Frankfurt/Main: VDR, 2004 